\title{
Fabrication of Diffractive Optical Elements in Polymers by 400-nm Femtosecond Laser Pulses
}

\author{
Wataru WATANABE ${ }^{* 1}$, Katsumi MATSUDA ${ }^{* 2}$, Satoshi HIRONO*2 ${ }^{* 2}$ and Hiroyuki MOCHIZUKI ${ }^{* 1}$ \\ ${ }^{*}$ Electronics and Photonics Research Institute, National Institute of Advanced Industrial Science \\ and Technology, \\ Umezono 1-1-1, Tsukuba, Ibaraki, 305-8568, Japan \\ wataru.watanabe@aist.go.jp \\ *2 Production Method Development Group, Omron Corp. \\ 2-1-2 Nishikusatsu, Kusatsu, Shiga 525-0035, Japan
}

\begin{abstract}
This report describes fabrication of volume gratings inside various matrix polymers using femtosecond laser irradiation at a wavelength of $400 \mathrm{~nm}$. We investigated the diffraction efficiencies of volume gratings with a period of $10 \mu \mathrm{m}$ by focusing $400-\mathrm{nm}$ laser pulses with a 0.1 -numerical aperture objective lens. We compared the polymers' diffraction efficiencies at $400 \mathrm{~nm}$ and $800 \mathrm{~nm}$. The largest refractive index change was induced in polymers with low density. The large refractive index change was attributed to its volume contraction based on its low density. DOI:10.2961/jlmn.2012.01.0011
\end{abstract}

Keywords: femtosecond laser, second-harmonic generation, filamentation, polymer, diffractive optics, micromachining

\section{Introduction}

Diffractive optical elements (DOEs) are attractive because of their compactness and high optical functions. The areas of DOEs' applications include beam shaping, materials processing, sensing, optical metrology, and lighting. Current techniques for fabricating DOEs are usually based on planar technologies. The integration of DOEs in monolithic bulk materials is expected to have high toughness and to be more suitable for device integration

Recently, the use of femtosecond laser direct writing to produce optical devices inside transparent materials has been expanding considerably [1,2]. Tightly focused femtosecond laser pulses can induce nonlinear absorption within the focal volume and can modify the indices of refraction of the materials permanently. This highly localized modification gives femtosecond laser micromachining a unique three-dimensional micromodification capability. Furthermore, femtosecond laser direct writing is an attractive technique because it enables one-step fabrication of three-dimensional optical devices.

Polymer materials are widely used for fabricating photonic components because of their various advantages such as low cost, ease of manufacture, and high transmission in the visible region. The use of intense femtosecond laser pulses enables the fabrication of threedimensional photonic structures such as memories [3-6], microchannels [7,8], waveguides [10-15], and gratings [16-28] in polymer materials. The widening integration of DOEs in bulk polymers is increasingly providing good mechanical stability and high integration density.

Fabrication of DOEs in polymer materials has been demonstrated using femtosecond lasers. To increase the diffraction efficiency of DOEs embedded in polymers, various fabrication conditions have been investigated, such as laser parameters [19,20], material types [24-27], and thermal treatment [28].

Filamentary modifications are crucial in the applications of DOE fabrication. Self-guiding of the beam created from ultrashort laser pulses, which is also called filamentation, occurs as a consequence of dynamic balance between Kerr self-focusing and defocusing effects in the electron plasma generated through the ionization process. Filament formation inside transparent solid materials engenders filamentary modifications, which are useful to fabricate volumetric optical elements in transparent materials because the shape of refractive index change is elongated along the optical axis [29-32].

Mochizuki et al. investigated refractive index change in various polymers using femtosecond laser pulses at a $1 \mathrm{kHz}$ repetition rate and a wavelength of $800 \mathrm{~nm}$ using a microscope objective with a numerical aperture (NA) of 0.1 . The thickness of gratings was $300 \mu \mathrm{m}$ using a filamentation process. The diffraction efficiency of a DOE in polymethylpentene (PMP) was an order of magnitude higher than those of other polymers [24]. The value of diffraction efficiency in PMP was $45.1 \%$, which was approximately an order of magnitude higher than the values of the other polymers, which were $0.3 \%-4.6 \%$. The refractive index change in PMP was estimated as $4.9 \times 10^{-4}$. Mochizuki et al. concluded that a low-density polymer with a large free volume seems to contract more easily than a denser material does. Baum et al. investigated the induction of refractive index change in poly(methyl methacrylate) (PMMA) using a 387 femtosecond laser [20 23]. This wavelength causes increased refractive index changes compared to the fundamental wavelength because of enhanced nonlinear absorption.

This report describes fabrication of DOEs inside various matrix polymers using femtosecond laser 
irradiation at a wavelength of $400 \mathrm{~nm}$. We investigated the induction of refractive index change in various matrix polymers by $400-\mathrm{nm}$ femtosecond laser pulses.

\section{Experimental system}

Figure 1 depicts the optical setup for fabrication of DOEs in bulk polymer materials. An amplified Ti:sapphire femtosecond laser $(800 \mathrm{~nm}$ wavelength, $1 \mathrm{kHz}$ repetition rate, 150 fs pulse duration) was frequency-doubled to 400 $\mathrm{nm}$. The pulse energy was attenuated by rotating a halfwave plate in front of a polarizer. A short-wave pass filter cut excluded laser pulses of a fundamental wavelength of $800 \mathrm{~nm}$. The 400-nm laser pulses were focused by a $5 \times$ microscope objective with a numerical aperture of 0.13 . The focal point was located $500 \mu \mathrm{m}$ below the sample surface. The energy was $3.5 \mu \mathrm{J} /$ pulse. The sample, which was mounted on a computer-controlled translation stage, was translated perpendicular to the laser beam propagation direction at a constant speed of $1 \mathrm{~mm} / \mathrm{s}$. The pulse energy at each sample surface was adjusted to $1000 \mathrm{~nJ}$ with an attenuator inserted between the laser equipment and microscope objective lens. Volume diffraction gratings of $8.0 \mathrm{~mm} \times 8.0 \mathrm{~mm}$ with a period of $10 \mu \mathrm{m}$ were fabricated in polymers by translating the samples with a twodimensional stage at $1 \mathrm{~mm} / \mathrm{s}$. We used a series of opticaluse polymers: PMMA (Acrylite L \#000; Mitsubishi Rayon Co. Ltd.), polycarbonate (PC, Panlite AD-5503; Teijin Chemicals Ltd.), cyclo-olefin polymer (COP, Zeonex 480; Zeon Corp.), PMP (TPX RT-18; Mitsui Chemicals Inc.), poly(methyl methacrylimide) (PMMI, Pleximid 8805; Evonik Industries), and polystyrene (PS, GPPS K27; PS Japan Corp.). The characteristics of all polymers were presented in earlier reports $[16,24]$.

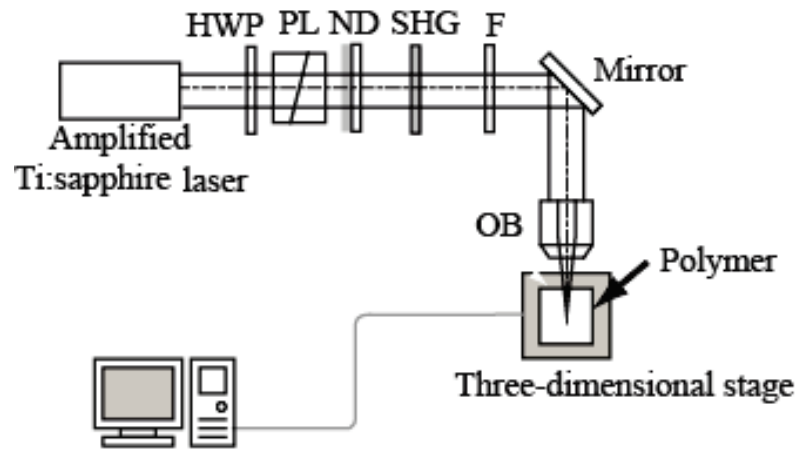

Personal computer

Fig. 1 Optical setup for fabrication of DOEs in polymers. HWP, half-wave plate; PL, polarizer; ND, neutral density filter; SHG, second harmonic generation crystal; F, shortwave pass filter, $\mathrm{OB}$, objective lens.

The diffraction efficiency was measured using a He-Ne laser at a wavelength of $632.8 \mathrm{~nm}$ so that the He-Ne laser beam incident upon the grating was diffracted at the firstorder Bragg diffraction angle. The intensity of each diffracted beam was measured using a power meter. We calculated the first-order Bragg diffraction efficiency $\left(\eta_{1}\right)$ as the ratio of the first-order diffracted beam intensity to the sum of all diffracted beam intensities.

The $Q$ value, a parameter used to judge the type of diffraction, is given by equation [34] as

$$
Q=\frac{2 \pi \lambda L}{n \Lambda^{2}}
$$

where $\lambda, L, \Lambda$, and $n$ respectively signify the measurement wavelength of the beam $(632.8 \mathrm{~nm})$, the diffraction grating thickness, grid interval, and the polymer refractive index. The diffraction grating period $(\Lambda)$ was observed from the sample surface using a transmission optical microscope. The grating thickness was observed from a direction perpendicular to the optical axis using an optical microscope. The grating thickness corresponds to the length of filamentary modification. A grating with a $Q$ value greater than 10 can be regarded as a thick grating showing Bragg diffraction properties. Because the diffraction grating area had no absorption and because the $Q$ value of the fabricated gratings was approximately 10 the refractive index change, $\Delta n$, was calculated as

$$
\eta_{1}=\sin ^{2}\left(\frac{\pi \Delta n L}{\lambda \cos \theta_{B}}\right)
$$

\section{Experimental results}

Figure 2(a) portrays a typical diffraction image of the embedded DOE in TPX. Figure 2(b) shows a top view of an optical image of the DOE fabricated in TPX. Periodic structures with a period of $10 \mu \mathrm{m}$ were fabricated inside TPX.

Table 1 shows first-order diffraction efficiencies of DOEs and estimated refractive index changes in various polymers. The grating thickness was $300 \mu \mathrm{m}$ by observation from with an optical microscope. The value of $\eta_{1}$ in PMP was $45 \%$, and that in COP was $34 \%$. The value of $\eta_{1}$ in PMMA was $11 \%$. The values of $\eta_{1}$ in PC and PMMI were, respectively, $2 \%$ and $3 \%$. The refractive index change in PMP was estimated as $4.9 \times 10^{-4}$. The value of $\eta_{1}$ and refractive index change in PMP were almost identical values to those induced with a femtosecond laser at a fundamental wavelength $(800 \mathrm{~nm})$ [24]. The refractive index change in PMMA was estimated as $2.3 \times 10^{-4}$. For PS the refractive index change is not detectable with an optical microscope. When the incident energy was increased, the refractive index change was not induced; scattering damage was produced in PS. 

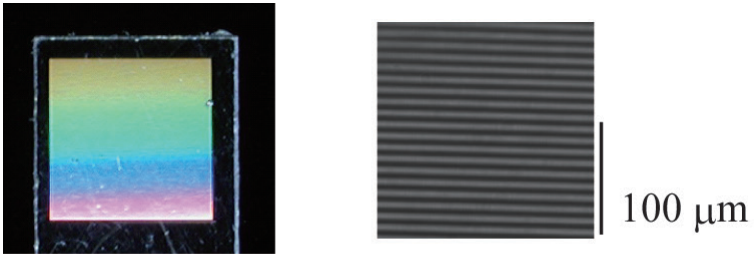

$8 \mathrm{~mm}$

(a)

(b)
Fig. 2(a) Diffraction image of the embedded DOE (b) optical image of the DOE fabricated in PMP.

Table 1 First-order diffraction efficiencies of DOEs and estimated refractive index changes. "Not clearly defined" in PS signifies that the modified region was too small to identify structural modifications.

\begin{tabular}{lcccccl}
\hline & PMMA & PC & COP & PMP & PMMI & PS \\
\hline $\begin{array}{l}\text { 1st order } \\
\text { diffraction } \\
\text { efficiency } \\
(\%)\end{array}$ & 11 & 2 & 34 & 45 & 3 & $\begin{array}{l}\text { Not } \\
\text { clearly } \\
\text { defined }\end{array}$ \\
\hline $\begin{array}{l}\text { Refractive } \\
\text { index } \\
\begin{array}{l}\text { change } \\
\left(\times 10^{-4}\right)\end{array}\end{array}$ & 2.3 & 1.0 & 4.2 & 4.9 & 1.2 & $\begin{array}{l}\text { Not } \\
\text { clearly } \\
\text { defined }\end{array}$ \\
\hline
\end{tabular}

\section{Discussion}

We compared the processes at $400 \mathrm{~nm}$ and $800 \mathrm{~nm}$. Figure 3 shows $\eta_{1}$ values of DOEs against material polymer densities. PMP had a density of $0.89 \mathrm{~g} / \mathrm{cm}^{3}$ [49] and exhibited the highest value of $\eta_{1}=45 \%$ in our experiments. COP had a density of $1.01 \mathrm{~g} / \mathrm{cm}^{3}$ and exhibited the high value of $\eta_{1}=34 \%$. PMMA, which had a density of $1.19 \mathrm{~g} / \mathrm{cm}^{3}$, exhibited a value of $\eta_{1}=11 \%$. The value was higher than that with laser pulses at a fundamental wavelength of $800 \mathrm{~nm}$. PC and PMMI had lower densities and diffraction efficiencies were lower than other polymers. For PS, the refractive index change was not detectable. The diffraction efficiency was not measurable.

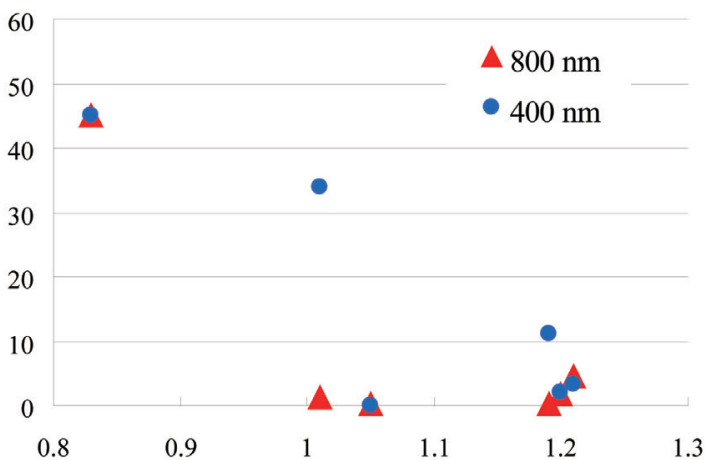

\section{Polymer density $(\mathrm{g} / \mathrm{cm})$}

Fig. 3 First-order diffraction efficiency of DOE as a function of the polymer material density. First-order diffraction efficiencies of DOE at a fundamental wavelength of $800 \mathrm{~nm}$ were referred from the literature [24].

The large refractive index change was induced in polymers with low density using a femtosecond laser at a fundamental wavelength of $800 \mathrm{~nm}$. This tendency was confirmed at a wavelength of $400 \mathrm{~nm}$ [24]. The density change is a possible explanation for the mechanisms. The physical mechanism responsible for the induction of refractive index change in PMMA has been investigated. Pulses of $40 \mathrm{fs}$ created phase gratings with refractive index changes of $\Delta n=5 \times 10^{-4}$ [18], and 85 fs pulses produced waveguides with $\Delta n=4.6 \times 10^{-4}$ [14]. The diffraction gratings described in an earlier report [9] showed up to $37 \%$ first-order diffraction efficiency. The results with a longer pulse duration of $180 \mathrm{fs}$ at a wavelength of $775 \mathrm{~nm}$ produced only small refractive index changes. In contrast, a frequency-doubled beam $(387 \mathrm{~nm})$ generated gratings with up to $40 \%$ first-order diffraction efficiency, corresponding to $\Delta n=4 \times 10^{-3}$ [19]. This discrepancy was attributed to the difference of focusing conditions and pulse durations of the laser pulse. Regarding the mechanisms in PMMA, the complete and partial separation of the side chain from the PMMA molecule [18,22] and tensile stress [14] were also responsible for inducing the refractive index change in PMMA. For PS, the refractive index change was not detectable at $400 \mathrm{~nm}$. Higher energy led to production of scattering damage. PS is a kind of fluorescent material with absorption band less than $300 \mathrm{~nm}$ arising from a benzene ring in its side chain. In multi-photon absorption inside PS, it is reasonable that the main deactivation of the excited state to the grand one seems to occur by mode of fluorescence emission rather than radical formation and photolysis leading to chemical reaction. The universal mechanism responsible for the induction of refractive index change in polymers is based on material density. 


\section{Conclusions}

In conclusion, we demonstrated the fabrication of DOEs in a series of optical polymers with femtosecond lasers at a wavelength of $400 \mathrm{~nm}$. Among the polymers, DOE formed inside PMP, which had the lowest density, and which showed the highest first-order diffraction efficiency value both at 800-nm and 400-nm femtosecond laser pulses. In polymers with low density, it is assumed to undergo contraction easily by femtosecond laser irradiation, resulting in a greater change in the refractive index. Direct micromachining using 400-nm femtosecond laser pulses is a potential method for three-dimensional integration of high efficiency DOEs in polymers.

\section{Acknowledgement}

The authors thank Y. Ozeki and K. Itoh from Osaka University for helpful discussion.

\section{REFERENCES}

[1] R. R. Gattass, and E. Mazur: Nature Photonics, 2, (2008) 219.

[2] K. Itoh, W. Watanabe, S. Nolte, and C. B. Schaffer: Mrs. Bulletin, 31, (2006) 620.

[3] K. Yamasaki, S. Juodkazis, M. Watanabe, H. B. Sun, S. Matsuo, and H. Misawa: Appl. Phy. Lett., 76, (2000) 1000 .

[4] O. Matoba, Y. Kitamura, T. Manabe, K. Nitta, and W. Watanabe: Appl. Phy. Lett., 95, (2009) 221114.

[5] Z. Nie, H. Lee, H. Yoo, Y. Lee, Y. Kim, K. S. Lim, and M. Lee: Appl. Phy. Lett., 94, 111912 (2009).

[6] J. Morikawa, A. Orie, T. Hashimoto, and S. Juodkazis: Appl. Phys. A - Materials Science \& Processing, 101, (2010) 27.

[7] K. Yamasaki, S. Juodkazis, S. Matsuo, H. Misawa: Appl. Phys. A 77, 371 (2003)

[8] D. Day, and M. Gu: Opt. Express, 13, (2005) 5939.

[9] L. Ding, R. Blackwell, J. F. Kunzler, and W. H. Knox: Opt. Express, 14, (2006) 11901.

[10]L. Ding, R. I. Blackwell, J. F. Kunzler, and W. H. Knox: Appl. Opt., 47, (2008) 3100.

[11] L. Ding, L. G. Cancado, L. Novotny, W. H. Knox, N. Anderson, D. Jani, J. Linhardt, R. I. Blackwell, and J. E. Künzler: J. Opt. Soc. Am. B, 26, (2009) 595.

[12] K. Ohta, M. Kamata, M. Obara, and N. Sawanobori: Proc. SPIE, 5340, (2004) 172.

[13] S. Sowa, W. Watanabe, T. Tamaki, J. Nishii, and K. Itoh: Opt. Express 14, (2006) 291.

[14] W. Watanabe, S. Sowa, T. Tamaki, K. Itoh, and J. Nishii: Jpn. J. Appl. Phys. Part 2, 45, (2006) L765.

[15]A. Zoubir, C. Lopez, M. Richardson, and K. Richardson: Opt. Lett., 29, (2004) 1840.

[16] S. Sowa, W. Watanabe, J. Nishii, and K. Itoh: Appl. Phys. A-Materials Science \& Processing, 81, (2005) 1587.

[17] L. Kuna, C. Sommer, E. Zinterl, J. R. Krenn, P. Pachler, P. Hartmann, S. Tasch, G. Leising, and F. P. Wenzl: Appl. Phys. A-Materials Science \& Processing, 93, (2008) 421.

[18]P. J. Scully, D. Jones, and D. A. Jaroszynski: J. Opt. A-Pure and Applied Optics, 5, (2003) S92.
[19] A. Baum, P. J. Scully, M. Basanta, C. L. P. Thomas, P. R. Fielden, N. J. Goddard, W. Perrie, and P. R. Chalker: Opt. Lett. 32, (2007) 190.

[20] A. Baum, P. J. Scully, W. Perrie, D. Jones, R. Issac, and D. A. Jaroszynski: Opt: Lett., 33, (2008) 651.

[21] S. J. Liang, P. J. Scully, J. Schille, J. Vaughan, M. Bwenyezzar, D. Liu, and W. Perrie: J. Laser Micro. Nanoeng., 5, (2010) 68.

[22]A. Baum, P. J. Scully, W. Perrie, D. Liu, and V. Lucarini: J. Opt. Soc. Am. B, 27, (2010) 107.

[23] A. Baum, S. De Nikola, S. Abdalah, K. Al-Naimee, A. Geltrude, M. Locatelli, M. Meucci, W. Perrie, P. J. Scully, A. Taranu, and F. T. Arecchi: Opt. Commun., 274 (2011) 2711.

[24] H. Mochizuki, W. Watanabe, R. Ezoe, T. Tamaki, Y. Ozeki, K. Itoh, M. Kasuya, K. Matsuda, and S. Hirono: Appl. Phy. Lett., 92, (2008) 091120.

[25] S. Katayama, M. Horiike, K. Hirao, and N. Tsutsumi: J. Polym. Sci. B, 40, (2002) 2800.

[26] S. Katayama, M. Horiike, K. Hirao, and N. Tsutsumi: Jpn. J. Appl. Phys., 41, (2002) 2155.

[27] C. Wochnowski, Y. Cheng, Y. Hanada, K. Meteva, S. Metev, K. Sugioka, G. Sepold, F. Vollertsen, and K. Midorikawa: J. Laser Micro. Nanoeng., 1, (2006) 195.

[28] S. Hirono, M. Kasuya, K. Matsuda, Y. Ozeki, K. Itoh, H. Mochizuki, and W. Watanabe: Appl. Phy. Lett., 94, (2009) 241122.

[29] K. Yamada, W. Watanabe, T. Toma, K. Itoh, and J. Nishii: Opt. Lett.26, (2001) 19.

[30] K. Yamada, W. Watanabe, Y. Li, K. Itoh, and J. Nishii: Opt. Lett., 29, (2004) 1846.

[31] K. Yamada, W. Watanabe, K. Kintaka, J. Nishii, and K. Itoh: Jpn. J. Appl. Phys., 42, (2003) 6916.

[32] W. Watanabe: Laser Physics, 19, (2009) 342.

[33] Plastic Processing Databook, 2nd ed., edited by the Japan Society for Technology of Plasticity (Nikkan Kogyo Shimbun, Tokyo, 2002), p. 2.

[34]H. Kogelnik: Bell Sys. Tech. J., 48, (1969) 2909.

(Received: May 21, 2011, Accepted: December 31, 2011) 